\title{
Diagnostic assays
}

\section{Erwin Schleicher}

Published online: 23 January 2009

(C) Springer-Verlag 2009

Rapid scientific and technological development has revolutionized the possibilities of diagnosing and treating diseases. Laboratory assays, together with methods based on physical principles, e.g. X-ray, ultrasound, nuclear magnetic resonance, etc., provide sensitive means of evaluating an individual's health. In most cases $(>50 \%)$ the results of laboratory tests contribute substantially not only to diagnosis but also to the aetiology of the diseaseto evaluating the acute state of the patient and searching for risk factors. Laboratory assays are important not only for diagnosis but also for prognosis - prediction of the course and possible outcome of a disease and of future diseases. Furthermore, an important future aspect of laboratory assays is the selection and monitoring of treatments with, e.g., very selective anticancer drugs. To fulfil these very different tasks a wide range of methods based on different chemical, biochemical, or physicochemical principles have been developed. These include methods using mass-, UVand visible spectroscopy, electrochemistry, immunology, nucleic acid hybridization, light scattering, and others. However, the choice of method not only depends on the nature of the analyte and the range of concentrations to be covered but also on the location where the analysis is performed.

While scientific and technological possibilities for sensitive, specific, precise, reproducible, and quantitative analysis of the analytes of interest are enormous, the development of a diagnostic device is driven by medical

\footnotetext{
E. Schleicher $(\bowtie)$

Clinical Chemist, Head of Clinical Chemistry Unit, University

Clinic of Tübingen,

Hoppe-Seyler-Str. 3,

72076 Tübingen, Germany

e-mail: Erwin.Schleicher@med.uni-tuebingen.de
}

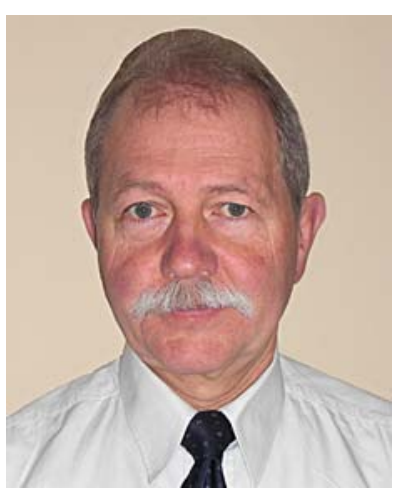

\begin{abstract}
Erwin Schleicher
is Professor of Clinical Chemistry and Head of the Clinical Chemistry Unit at the University Clinic Tübingen, Germany. His major interests focus on the molecular mechanism of insulin signal transduction and its impairment, leading to insulin resistance and type 2 diabetes, and on the pathogenesis of diabetic nephropathy. In clinical chemistry his major interests are the search for laboratory results indicating the development of dia-
\end{abstract} betes and its complications and on the miniaturization of diagnostic assays. His scientific work is published in more than 200 contributions.

need. This includes the choice of marker and the location where the analysis is performed, i.e. near the patient (point of care), in a small laboratory, or in a centralized large laboratory. Furthermore, for development of an analytical device it is important to define the user, i.e. layman/patient (e.g. for glucose measurements at home), medical staff (emergency unit, ambulance, doctor's office), or specialized staff in centralized laboratories. This is not only important for the training of the users but also for legal aspects.

Whereas in the past, particularly in hospitals, organizational and administrational issues were important, now the individual patient is moving more into the centre of attention. The adoption of a more patient-centred approach to care is also seen as having an effect on the hospital process, and as being more desirable for the patient. Thus, the services should come to the patient rather than the reverse. However, because of the highly sophisticated technical approaches in X-ray, NMR, and laboratory medicine these diagnostic services are moving further away from the patient to highly specialized centres. The 
laboratory, in particular, has made enormous efforts to come closer to the patient by establishing small specialized units near emergency rooms and intensive-care units and by developing devices for POCT. However, because of the plethora of different markers with different measurement ranges needed in laboratory testing, currently available POCT devices are very limited in the amount of markers they can measure.

This special issue on "Diagnostic Assays" offers the analytically orientated reader both an insight into the current status of laboratory assays with different applications in the still emerging field of laboratory tests. Wherever applicable the particular focus is on POCT.

The first two contributions describe the analytical procedures available for specific protein determination using either microarrays or for POCT. The principle of biosensor analysis is demonstrated and further illustrated by an application to determination of auto-antibodies. The emerging field of capillary electrophoresis coupled to mass spectrometry is described in principle, and application to urinary proteome analysis is critically covered and is illustrated in the cover motif of this issue. With this effective analytical procedure not only renal and urological disorders but also systemic diseases such as cardiovascular or infectious diseases may be detected and monitored. Blood-group typing is a special field in which screening methods and also bed-side tests need to be performed. The different current approaches and future trends are critically reviewed in this contribution.

The next three articles focus on POCT in three already widely used areas. A growing application with an economic impact of importance similar to that of blood-glucose testing is the self testing and self-management of a patient's coagulation status when chronically treated with an oral anticoagulant. Despite enormous efforts, currently available devices are still not satisfactory, because severe and even fatal events still occur much too often. A major unresolved issue is the problem of standardization of the assays in use, which are based on very different measurement principles.
The most widely used laboratory data at the patient's site are cardiac markers and biomarkers indicating inflammation. From both contributions it is clear that:

1. quantitative rather than semi-quantitative analysis of a marker is required, and

2. a combination of markers is needed for evaluation of the state of the patient.

A totally different diagnostic field is screening for inborn errors of metabolism and for endocrinopathies. This includes nationwide screening of all neonates for defects which can be treated when detected within the first week(s) of life. This objective is particularly challenging, because the methods need to be sensitive and specific, e.g. mass spectroscopy, and very inexpensive, because of the large number of tests. It should be noted that more than $99.9 \%$ of the individuals tested are healthy.

The advantages and disadvantages of POCT versus centralized laboratory testing are critically discussed, with particular emphasis on the time needed, in an article focusing on these general considerations.

Finally, analytical validation of a device for cancer biomarker discovery using multiplexed gene expression profiling is presented.

In brief, this issue on diagnostic assays enlightens the multifaceted field of laboratory medicine showing the very different markers measured, the techniques applied to achieve the sensitivity, specificity, and variation needed, different locations of application, and users from layman to laboratory specialist. Furthermore, the significant impact of this emerging field on the growing market of POCT can be envisaged. However, economic issues, a major topic of our field, is not covered, because this is very heterogenic, must be analyzed for each marker, varies from country to country, and involves health-care policies and thus exceeds the scope of this issue. In addition ethical problems arising from the possibility of recognizing the susceptibility of an individual to disease(s) in the future are also far beyond the scope of this issue. 